\title{
Improved Parallel DBSCAN Algorithm Based on Radix Sort
}

\author{
Manish Bhardwaj' and Dattatraya Adane ${ }^{2}$ \\ ${ }^{1}$ Ph.D. Scholar, Computer Science and Engineering Department, Shri \\ Ramdeobaba College of Engineering and Management, Nagpur (MS), India. \\ ${ }^{2}$ Department of Information Technology, Shri Ramdeobaba College of \\ Engineeing and Management, Nagpur (MS), India.
}

\section{ABSTRACT}

DBSCAN is one of the main density-based clustering algorithms and can find arbitrary shaped clusters and eliminate noise data. However, DBSCAN has a Quadratic time complexity, making it difficult in application with a large data set. Parallelization, DBSCAN to improve its performance is a challenging task because of OpenMP or MPI environments, there is no guarantee of proper load balancing and fault tolerance. We present the improved parallel DBSCAN algorithm using the OpenMP environment based on the Radix sort to solve the problem of load balancing. Shared memory programming is used to divide the task and then uses the Radix sort method to select data points for DBSCAN for clustering. With a large number of data points original DBSCAN unable to compute the clusters with limited memory, this improved parallel DBSCAN can able to handle the same. Experiments show that this improved method obtains better results with original DBSCAN while shows significant improvement in computation time requirement.

KEY WORDS: PARALLEL CLUSTERING, DBSCAN, SHARED MEMORY PROGRAMMING, PARALLELIZATION, RADIX SORT, OPENMP.

\section{INTRODUCTION}

Clustering divides the data into groups that are useful or meaningful or both. Data objects that are similar to each other within the same cluster and those are dissimilar to the objects in another cluster. Clustering based on density can discover the arbitrary shape clusters and able to remove the noise. Clustering has a wide range of application areas such as a statistic, information retrieval, pattern recognition, biology, and data mining. DBSCAN is presented in 1996. One of the main densitybased clustering algorithm. DBSCAN is a density-based

\section{ARTICLE INFORMATION}

*Corresponding Author: manish.bhardwaj1sept@gmail.com Received 17th Oct 2020 Accepted after revision 28nd Dec 2020 Print ISSN: 0974-6455 Online ISSN: 2321-4007 CODEN: BBRCBA

Thomson Reuters ISI Web of Science Clarivate Analytics USA and Crossref Indexed Journal

\section{Clarivate crossef}

NAAS Journal Score 2020 (4.31)

A Society of Science and Nature Publication,

Bhopal India 2020. All rights reserved.

Online Contents Available at: http//www.bbrc.in/

Doi: http://dx.doi.org/10.21786/bbrc/13.14/39 method that finds clusters according to a density-based connectivity analysis. DBSCAN calculates the density by counting the number of points in a fixed-radius neighborhood and considers two points as connected if they lie within each other neighborhood. Core points are points with a dense neighborhood. Border points are points that belong to a cluster but whose neighborhood is not dense. Core points are points with a dense neighborhood. Border points are points that belong to a cluster but whose neighborhood is not dense, Noise can be defined as the set of points in the database not belonging to any of its clusters [Martin Ester, 1996].

Different Parallel approaches are being used to reduce the load balancing problem faced by Clustering algorithms. Multiple Parallel DBSCAN algorithms are created and try to break the sequential processing. Most of the Parallel implementation tries to solve the following problems: Load Balancing: The DBSCAN execution time varies due to skewed data distribution to different processing elements. Some of them lightly loaded the other are heavily loaded. 
1. Data Split: A region split approach make the work more complicated, if we consider the data points distribution in each sub- region. It increases the overall time requirement of computation.

2. Expensive Merging: The sum of data points processed in sub-region is always greater than the overall sum of all data points. This increase overall execution time.

It is observed that DBSCAN computation is performed sequentially. To take the advantage of multiple processors (core) available in today's system. Fist the sequentiality is required to break. Data structure like $\mathrm{R}$-tree, $\mathrm{R}^{*}$, Disjoint set data structure can be used to improve the performance. In our approach, we use a shared-memory programming API called OpenMP to take the advantage of Fork-Join Model to perform the independent subtask. The Radix sort approach is used to handle load balancing among different threads [ Guoping Xiao, 2014]. The data points are sorted by their attribute values which are used for clustering. This is the key of all partition allocated to the different thread are having proper load balancing and points which belong to similar clusters. OpenMP API contains a good number of the parallel directive and constructs which help to perform the task in parallel.

First of all the parallel blocks of codes are identified after that proper parallel constructs are used to operate in parallel. In Fork-Join model Task is divided into multiple subtasks and this subtask is independently performed by different threads. Once all threads complete their subtask the sub results are combining by a single thread to compute the overall result. Shared memory programming required proper synchronization among different thread otherwise the result calculated by the thread may not be acceptable [Barbara Chapman, 2008]. This paper is organized as following: Section II, briefly introduce the related work, Section III, presents the improved parallel DBSCAN, Section IV, explained the Experiments and Evaluation results, Section V, we conclude our work .

Related Work: A number of parallel algorithms are proposed to solve the load balancing problem associated with DBSCAN. In most of the methods they try to achieve the better speedup using parallelization. They used different platform to implement their code, most of the cases they take the advantage of data structure. Different partitioning techniques are used for better load balancing. Stefan Brecheisen et al. they cited two limitations of DBSCAN: first, it is facing difficulty while running it on a very large databases duo to limited scalability; second, most of the available implementation not deal well with heterogeneous data sets where the similarity between item is represented by arbitrary complex functions. They target both problems and proposed an approximated, scalable, distributed DBSCAN implementation which can handle arbitrary data items and can able to handle symmetric distance function.

The algorithm is based on vertex-centric design compute a neighbor graph, a distributed data structure use to find a neighborhood of data points. Computations of clusters are based on the neighbor graph so the name is NG-DBSCAN. This DBSCAN is implemented in Spark, suitable to enable distributed vertex-centric. Graph-Based Clustering takes as input graphs and edges represent item similarity [Stefan Brecheisen, 2006].

Domenica Arlia and Massimo Coppola first addressed the performance of region queries by applying a simple replication approach. They absorb most of the processing time is utilized by region queries. The parallel implementation used the Mater Slave approach. The master module performs cluster assignment and the Slave module answers neighborhood quires by using $\mathrm{R}^{*}$-Tree. Two problems associated with Parallel implementation are the separation of spatial and labeling information. This can be avoided by nurturing local information by Slaves. The Slaves utilize it to eliminate unessential results. The parallel version of the code has been run on the cluster of 10 personal computers and uses the two data sets. This shows the scaled-up property of the code. With the help of the Parallel version of the code, 6 times speedup is achieved by using 8 numbers of salves [Domenica Arlia, 2010].

Min Chen et al. they cited that DBSCAN computational cost is expensive when the database is huge. They present P-DBSCAN, a novel parallel version implemented in a distributed environment. It uses practically efficient and optimal Priority R-Tree. The database is partition into several parts; each computational node builds a PR-tree and can perform clustering independently; Sub results will be required to aggregate into the final result. They show the comparison between P-DBSCAN and original DBSCAN, they found that P-DBSCAN is superior to the DBSCAN in terms of quality of the result, Speedup achieved, and Scalability [Min Chen, 2010].

Md. Mostofa Ali Patwary et al. they cited access sequential way of DBSCAN, to break it they present a new Parallel DBSCAN algorithm (PDSDBSCAN) which takes the advantage of disjoint-set data structure. The steps involved in their approach are as follows: The algorithm first creates a single node tree for every dataset; after that disjoint-set data structure is used to merge the trees belongs to the same cluster until all clusters are not discovered. Merging is performed arbitrarily and this is the key approach. PDSDBSCAN shows better scalability and load balancing. They implemented their algorithm by using the Shared Memory and Message passing approach. OpenMP implementation used 40 numbers of processing elements and MPI they used 8192 numbers of processing elements and achieved 25.97 times and 5765-time speedup respectively [Md. Mostafa Ali Patwary, 2012].

Tatsuhiro Sakai et al. they proposed a master-worker model using data parallelism. A spatial cluster can be extracted independently of other spatial clusters by DBSCAN at the data level. Data Partitioning is applied to geo-social databases by dividing the overall database into two or more partitions by using grid partitioning. The proposed model uses the task pool to distribute the loads. Processing of spatial clustering for a partition 
associated with a complex grid is referred to as a task. The master thread manages the task pool. Clustering is performed by each worker after they obtain a task from the task pool. If workers complete processing and no more tasks available in the task pool then the work is completed [Tatsuhiro Sakai, 2016].

Yinghua Lv et al. they proposed a solution. Firstly, they improve the locality sensitive hashing method to perform a fast query of nearest neighbors. Secondly, nearest neighbors and reverse neighbors are taken into the consideration to construct influence space of each object. In the final step, this influence space is utilized to differentiate border and noisy objects. In the improved algorithm IS-DBSCAN, they use the influence space (IS) to obtain a better estimation of the neighborhood density distribution. This method is symmetric in nature and able to identify any objects [Yinghua Lv, 2016]. Hawan Jun Song and Jae-Gil Lee propose a Random PartitioningDBSCAN (RP-DBSCAN) that takes benefits of the random split strategy and removes the same split restriction. Two approaches are used to solve the technical problem associated with partitioning. The first is called a pseudorandom technique and the second is a two-level cell dictionary. This is basically a two-level tree. A cell is represented by the first level of a node and the second level shows the sub. Every node encodes the number of points in each cell and its position. They proved by experiment that the overhead is solved to some extent [Hawan Jun Song, 2018].

Gan and Tao first cited that the worst-case complexity of DBSCAN is 0 (n2) proven. They showed that till 2-dimensional data DBSCAN can run in $0(n \log n)$ and quickly start showing quadratic behavior with high dimensional data. They proposed DBSCAN++, a modified DBSCAN which only required computing the densities for a chosen subset of points. DBSCAN++ is based on the observation that, it is only required to compute the density estimates for a subset $m$ of the $n$ data points where $\mathrm{m}$ is much smaller than $\mathrm{n}$. It is sufficient to construct the clusters properly. To choose $\mathrm{m}$ points uniform and greedy k-center-based sampling is used. The resulting procedure shows $\mathrm{O}(\mathrm{mn})$ worst-case runtime. This is proved by the experiments they have done.

Improved Parallel DBSCAN: To speed up the performance of DBSCAN, we proposed the improved parallel DBSCAN including two approaches (1) OpenMP API (2) Radix Sort approach.

(1) OpenMP API:The application programming interface OpenMP is Open Multiprocessing shared-memory multiprocessing programming supported by $\mathrm{C}, \mathrm{C}++$, and Fortran. It is based on a model called Fork-Join. This is the key approach to break the computation sequentiality associated with DBSCAN. OpenMP supports parallel directive and constructs, like parallel loop construct which is help to perform the computation in parallel. Task decomposed in multiple sub tasks and performed by multiple threads, run in synchronized manner. If task is not divided into sub task it required to perform atomically by single thread.

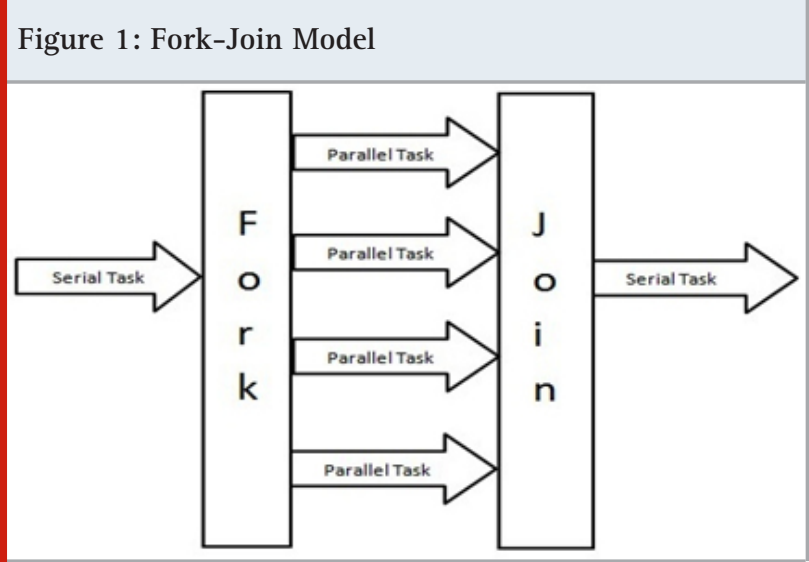

(2) Radix Sort Approach: OpenMP implementation of DBSCAN using parallel constructs in which multiple threads are performing the computation. To take the advantage of this approach data set is dividing into multiple partitioned. For proper load balancing of thread, the data partitions are sorted so the points belong to the same clusters are allotted to a specific thread. This way the global clusters are computed by using multiple local clusters. OpenMP breaks the sequentiality of DBSCAN and Radix sort help for proper load balancing among threads. Proper numbers of threads are required to create otherwise synchronization takes more time. This approach takes the advantage of both OpenMP and Radix Sort.

Experimental Analysis: Data sets: We use ten KEEL data sets named Quake, House16, Layout Histrogram, Color Histrogram, Color Movement, Transaction10k, Transaction20k, Transaction30k, Transaction40k, and Transaction50k to compare the performance analysis in term of execution time. In tables presents Name of data sets Name, Number of data points N, Execution Time T, Number of Clusters C, Noise Points NP, Minimum Number of Points M, Radius E and Dimension of the Data Sets represented by $\mathrm{D}$.

Evolution System Hardware: We use the system with following characteristics a) Processor: Intel Core i3 processor @2.53GH b) Ram: 4 GB c) Operation System: Ubuntu 18.04LTS d) Program: C++ using OpenMP API. In order to evaluate improved parallel DBSCAN clustering algorithm. GCC compiler is used to executer the Parallel implementation of Programs. The Experimental Data Sets are chosen from the KEEL Data Sets Repository. Improved parallel DBSCAN and original DBSCAN are implemented.

The analysis is performing based on execution time and other factor such as No. of clusters and noise point find by both programs. Execution time is used for defining speedup of improved parallel implementation of DBSCAN. Table I and Table II shows when data sets size 
is small both of the program execution time relatively equal. But original DBSCAN algorithm unable to handle data sets with large number of data points such as Transaction30k, Transaction50k, Transaction70k and
Transaction90k. Which is represented in Table I by "?”. Table II is shows the Improved DBSCAN Computation of the Clusters. Improved Parallel DBSCAN can able to calculate clusters for all and its shows 26 times speedup for Transaction 10k data set.

\begin{tabular}{|c|c|c|c|c|c|c|c|c|}
\hline S. No & Name & $\mathrm{N}$ & $\mathrm{T}$ & $\mathrm{C}$ & NP & M & $\mathrm{E}$ & $\mathrm{D}$ \\
\hline 1 & QUAKE & 2185 & 0.023812 & 3 & 65 & 10 & 10 & 2 \\
\hline 2 & HOUSE16 & 22804 & 10.82550 & 13 & 3316 & 10 & 10 & 2 \\
\hline 3 & LAYOUT HISTOGRAM & 66651 & 0.029158 & 3 & 13 & 10 & 10 & 2 \\
\hline 4 & COLOR HISTOGRAM & 68076 & 0.028135 & 2 & 13 & 10 & 10 & 2 \\
\hline 5 & COLOR MOVEMENT & 68053 & 0.028730 & 1 & 1 & 10 & 10 & 2 \\
\hline 6 & TRANSACTION10K & 120433 & 1034.28 & 4 & 7 & 10 & 10 & 2 \\
\hline 7 & TRANSACTION30K & 284290 & $?$ & $?$ & $?$ & 10 & 10 & 2 \\
\hline 8 & TRANSACTION50K & 475655 & $?$ & $?$ & $?$ & 10 & 10 & 2 \\
\hline 9 & TRANSACTION70K & 665476 & $?$ & $?$ & $?$ & 10 & 10 & 2 \\
\hline 10 & TRANSACTION90K & 855373 & $?$ & $?$ & $?$ & 10 & 10 & 2 \\
\hline
\end{tabular}

Table 2. Improved Parallel DBSCAN Computation of Clustering

\begin{tabular}{|l|c|c|c|c|c|c|c|c|}
\hline \multirow{2}{*}{ S. No } & Name & N & T & C & NP & M & E & D \\
\hline 1 & QUAKE & 2185 & 0.021812 & 3 & 65 & 10 & 10 & 2 \\
\hline 2 & HOUSE16 & 22804 & 8.238900 & 13 & 3316 & 10 & 10 & 2 \\
\hline 3 & LAYOUT HISTOGRAM & 66651 & 0.764452 & 3 & 13 & 10 & 10 & 2 \\
\hline 4 & COLOR HISTOGRAM & 68076 & 0.906587 & 2 & 13 & 10 & 10 & 2 \\
\hline 5 & COLOR MOVEMENT & 68053 & 1.031820 & 1 & 1 & 10 & 10 & 2 \\
\hline 6 & TRANSACTION10K & 120433 & 39.6971 & 4 & 7 & 10 & 10 & 2 \\
\hline 7 & TRANSACTION30K & 284290 & 168.030 & 4 & 7 & 10 & 10 & 2 \\
\hline 8 & TRANSACTION50K & 475655 & 493.144 & 4 & 7 & 10 & 10 & 2 \\
\hline 9 & TRANSACTION70K & 665476 & 1014.14 & 4 & 7 & 10 & 10 & 2 \\
\hline 10 & TRANSACTION90K & 855373 & 1735.37 & 4 & 7 & 10 & 10 & 2 \\
\hline
\end{tabular}

\section{CONCLUSION}

The experimental analysis clear that it is convenient to adopt the Parallel approach based on OpenMP. In Parallel approach all processing elements. The Parallel approach required less time as compared to the Sequential approach. In this improved parallel DBSCAN, it uses the Radix sort, which helps to divide the overall data set into a small number of the partition. Each processor is working with different partitions and computing clusters. This way better speedup is achieved. It is clear from the experiment that the original DBSCAN implementation unable to calculate clusters for data sets Transaction30k, Transaction50k, Transaction70k, and Transaction90k with available primary memory. Improved Parallel DBSCAN able to compute the clusters and with Transaction 10k its shows 26-time speedup.

There are some directions for future research:

1. The same approach can be used with other density based clustering algorithms with some changes.
2. This approach can be modified for MPI programming environment for better speedup and for large data sets.

\section{REFERENCES}

Alessandro Lulli, Matteo Dell Amico, Pietro Michiardi and Laura Ricci, "NG-DBSCAN: Scalable Density-Based Clustering for Arbitrary Data", ACM Proceedings of the VLDB Endowment, Volume 10, 2016, pp 271-277.

Barbara Chapman, Gabriele Jost and Ruud Van Der Pas, Using OpenMP Portable Shared Memory, MIT Press publication, 2008, pp 20-200.

Dianwei Han, Ankit Agrawal, Wei-keng Liao and Alok Choudhary, "A Novel Scalable DBSCAN Algorithm with Spark”, Parallel and Distributed Processing Symposium Workshop, 2016, pp 1-5.

Domenica Arlia and Massimo Coppola, "Experiments in Parallel Clustering with DBSCAN", Lecture Notes in 
Computer Science, 2010, pp 1-6.

Guoping Xiao, M. Martina, G. Masera and G. Piccinini, "A Parallel Radix-Sort Based VLSI Architecture for Finding the First \$W\$ Maximum/Minimum Values, IEEE Transactions on Circuits and Systems, Volume 61,no.11,2014,pp 890-894.

Hawan Jun Song and Jae-Gil Lee, "RP-DBSCAN; A Superfast parallel DBSCAN Algorithm Based on Random Partitioning”, ACM Sigmod Conference, 2018, pp 1-5.

Martin Ester, Hans-Peter Kriegel, Jorg Sander and Xiaowei Xu," Density-Based Algorithms for Discovering Clusters in Large Spatial Databases with Noise", Proceedings of the Second International Conference on Knowledge Discovery and Data Mining, 1996, pp 1-7.

Md. Mostofa Ali Patwary, Diana Patsetia, Ankit Agrawal, Wei-keng Liao, Fredric Manne and Alok Choudhary., "A New Scalable Parallel DBSCAN Algorithm Using the Disjoint-set Data structure", Proceedings of the International Conference on High Performance Computing, Networking, Storage and Analysis”, 2012, pp 1-8.

Michael Gowanlock, David M. Blair and Victor Pankratium, "Optimizing Parallel Clustering Throughput in Shared Memory" IEEE Transaction of Parallel and Distributed System, 2017, pp 1-7.
Min Chen, Xue Dong Gao and Hui Fei Li, "Parallel DBSCAN with Priority R-tree” 2nd International Conference on Information Management and Engineering, ICIME, 2010, pp 1-5.

Stefan Brecheisen, Hans-Peter Kriegel and Martin Pfeifle, "Parallel Density-Based Clustering of Complex Objects”, 10th Pacific-Asia Conference on Knowledge Discovery and Data Mining, 2006, pp 1-5.

Tatsuhiro Sakai, Keiichi Tamura, Kohei Misaki and Hajima Kitakami, "Parallel Processing for DensityBased Spatial Clustering Algorithm using Complex Grid Partitioning and Its Performance Evaluation”, International Conference of Parallel and Distributed Technology Application, 2016, pp 1-7.

Xultn Jun Huang and Minaghui Qin, "MR-DBSCAN: An Efficient Parallel Density-Based Clustering Algorithm using MapReduce", 17th International Conference on Parallel and Distributed System, IEEE, 2011, pp. 1-5. Vu Viet Thang and D.V. Pantiukhin, "A Hybrid Clustering algorithm: the Fast DBSCAN", International Conference on Engineering and Telecommunication, 2015, pp 1-5.

Yinghua Lv, Tinghua Ma, Meilli Tang Yuan Tain, Jie Cao, Abdullab Al-Dhelaan and Mznah Al-Rodhaan, "An Efficient and Scalable Density-Based Clustering Algorithm for Datasets with Complex Structure" Journal of Neurocomputing, Volume 171, 2016, pp 222-228. 